\title{
Research of Psychological Intervention of College Students' Internet Addiction Disorder
}

\author{
Zhongxia Cai \\ Marine college of Shandong jiaotong university, Weihai, Shandong,China
}

\begin{abstract}
Keywords: College Students, Internet Addiction Disorder, Psychological Intervention, A disciplinary offence, Countermeasures
\end{abstract}

\begin{abstract}
Internet has become an integral part of college students ' learning life, the network has penetrated into all aspects of daily life of college students. From the network, they continued to draw all kinds of nutrition, exposure to cutting-edge knowledge of this subject for the latest information. But overuse of some college students to network, form a network of heavy reliance on their study, life and physical and mental health has been severely affected, and many serious consequences arising therefrom. Internet addiction and its negative effects have caused widespread concern in the University and even the whole society. The study would help to improve the college students ' Internet addiction awareness and attention, perfect the control system of college students ' Internet addiction intervention for improving college students ' psychological health of Internet addiction disorder, has positive significance in promoting the all-round development of college students.
\end{abstract}

\section{Introduction}

The computer network is one of the most remarkable achievements in the development of 20th century science and technology, computer network occurred rapid development as we enter the information age and has laid a solid foundation. Network to its interactive and timely openness, anonymity and features to attract a large number of user groups, and gradually infiltrated into daily learning, life and work, influence people's lifestyles, ideas, values and mental health.

Network in China appear relatively late, but rapid development, according to the China Internet network information center (CNNIC), the 28th statistical report on Internet development in China, Internet has been ranked first in the world. At present, the skilled use of the network of college students has been regarded as a matter of course, familiar with network knowledge and with a certain degree of college students in Internet skills has become a fundamental requirement. Popularization of network on college campuses, on the one hand broaden students ' horizons, changed the traditional teaching mode of education, broaden students ' knowledge acquisition channels, improving students ' knowledge structure and reserve. The other hand, as the technology of "double edged sword" in help young students at the same time, improper use of network to college students also bring about many negative impacts. Due to the physical and mental development of college students still had not yet full-fledged stage, they do not have high levels of cognitive and self-control, in the experience of the network in the course of the world, easy to be attracted to the negative aspects of the Valley in the network, part of college students indulging in network world, lead to Internet addiction.

According to existing of survey results, students network addiction groups take shape, network addiction led to students physical and mental health, and social contacts, and learning, aspects of problem has increasingly highlight, students network addiction problem on University thought political education proposed has new of challenge. network addiction on students of physical and mental health caused has huge of effect, directly threat to university students of health growth and campus harmony stable, on achieved thought political education of target, and training construction Socialist career qualified talent of task caused has impact. College students ' Internet addiction has become the ideological and political workers of a problem. How to tell if a college student suffering from Internet addiction and its extent, and built according to the actual situation of college students ' Internet addiction disorder control system, particularly the psychological intervention mechanism, has become the ideological and political education needed to address the major issues. 


\section{An overview of Internet addiction}

"Internet addiction" is from the United States first proposed by psychiatrist Dr Goldberg, when using the Internet addiction disorder this phrase, followed by different scholars to name certain differences in the expression of Internet addiction, currently a representative reference: Internet addiction disorder, Pathological Internet use, pathological Internet use, network Act, Online Addiction, and so on.

World Health Organization defines addiction addiction as a result of the excessive use of the network as a result of chronic or recurring obsession with status and irresistible desire to use again, and can produce phenomena such as withdrawal, tolerance reaction, for the pleasure of the Internet there are psychological and physical dependence.

In November 2008, China's first Internet addiction diagnostic criteria, by experts, this concept of Internet addiction disorder is expressed as: individuals repeated excessive Internet use, social function of a psychiatric disorder, have a strong desire for the network again using, stopping or reducing the use of withdrawal phenomena.

On the concept of addiction vary, each definition there are some deficiencies, but the content is basically the same of Internet addiction, mainly in the uncontrolled use of individual networks, individual physical and mental health damage, and so on. These ideas, concept of Internet addiction can be expressed as follows: in the presence of non-addictive substance, individuals have no control over their online behavior and excessive Internet use for a long time, resulting in impaired individual physiological, psychological and social function of the State.

A. Types of network addiction

Internet addiction based on different criteria, you can do different types. Internet addiction is addiction caused by the network, according to the different addictive Internet behavior, Internet addiction can be divided into Internet pornography addiction, Internet addiction, impulse Internet addiction, addiction, addiction to computer games five types of information overload.

Cyber sexual addiction refers to Internet addiction to online content associated with a porn addiction, mainly for regular browsing pornographic Web sites, look for associated erotic pictures, erotic texts, adult movies as well as ongoing sexual chat, and so on. Network porn on youth physical and mental health of negative effect especially far-reaching, from students of physical and mental development of angle, while they has gradually into has sex mature period, they on sex of curiosity to far above other age stage of netizens, on the in reality life in the, by existing education and traditional concept of effect, they hard through normal of way get related knowledge, and network porn of exists meet has they adventures of psychological.

Internet relationship addiction is Internet addicts indulge in virtual network communication, like through QQ, MSN and Internet communication tools such as chat rooms and meet some friends, build your own interpersonal circle, their interest in real interpersonal weakened network gradually replaced the reality of interpersonal relationships.

Impulse Internet addiction is due to excessive Internet users shop online, online gambling and other activities as a result of.

Information overload Internet addiction disorder, this type of compulsive Internet addiction is mainly for Internet users browse the Web to collect information and data not related to real life.

Computer game addiction, which is one of the most common types of Internet addiction, and most types of Internet addiction for teenagers. Computer games including games, single games, online role playing games, and more. Computer games make young people access to a virtual sense of achievement at the same time, their cognitive model, behavior and physical and mental health has caused serious damage.

To classify Internet addiction, contribute to a deeper knowledge and understanding of Internet addiction phenomenon, particularly network made by the Division, so that we can analyze network behavior motivation needs to find individual causes of Internet addiction, and lay the Foundation for selecting intervention point, interventions. 
B. The main features of Internet addiction

As a behavioral addiction, Internet addiction, but it also has some of the characteristics of drug addiction, and synthesize existing research, characteristics can be summarized as the prominence of Internet addiction, tolerance, withdrawal, conflict and repetition, and so on.

Salience, it refers to the Internet addiction disorder of thoughts, feelings and behavior have been significantly controlled by the Internet this activity, Internet account for Internet addiction in life of a lot of time.

Tolerance, it reflects the Internet addiction dependency on network and continuously improve, specifically Internet addiction Internet frequency and increasing time online, Internet addicts to obtain satisfaction with the same need to increase Internet access time.

Withdrawal, if it refers to Internet addiction is not online or when forced into the net in the Middle, will have a negative psychological mood, accompanied by adverse physiological responses.

Conflicts, conflicts of Internet addiction is mainly manifested in the following three aspects: first of all Internet activity has had a conflict with individuals ' physical and psychological development followed in the study on the contradiction between Internet access and individual last is also reflected in the relationship between individual and social environment with others. Conflict characteristics reflect the network behavior of Internet addiction the objective effects.

Repeatedly, this means that Internet addicts after the treatment or after the self-regulation, ring in addition to Internet addiction or reduce the dependency on network, but if there are no further consolidate, in due time, it will once again have Internet addiction, or to the extent of reliance placed on the network before the intervention.

\section{Investigation and analysis of Internet addiction among college students}

The study of Shandong University, Harbin Polytechnic University, Shandong Jiaotong University and other college students as participants in the survey were selected from 2010 to 2013, more than four-year amounts to more than 500 students, respondents involved in arts, science, engineering, and other disciplines. The questionnaire uses a secret, random sampling in the College, students collectively or individually to answer, fill in the complete return of the questionnaires on the spot. Eventually, sent out questionnaires, 510, recovered after finishing, removing valid questionnaires, total effective questionnaire 485 , effective recovery of $95.1 \%$.

In accordance with the criteria of this scale, data were obtained from statistics, to 485 Internet addiction disorder of undergraduates were determined. According to statistics, 75 students were identified as Internet addicts, $15.46 \%$ per cent of all respondents. Judging from the results of this survey, groups of college students ' Internet addiction scale can not be overlooked, caused widespread concern in the University and society.

More specifically, in order to master the Internet addiction of college students, clear the individuals dependent on networks, provide the basis for targeted psychological interventions, this study of college students ' Internet addiction group made further analysis, define different levels of Internet addiction college students ' Internet addiction. According to the analysis, Internet addiction disorder among college students, and to a lesser extent accounted for the vast majority of Internet addiction, accounting for $73.33 \%$ of the total, medium degree of addiction accounted for $24 \%$ of college students ' Internet addiction as a whole group, while $2.67 \%$ of Internet addiction among college students has reached a serious level.

Based on the analysis of college students ' Internet addiction scale and extent of, in this study, two groups of college students Internet addiction college students and non-learning, major network, compare the richness of Internet activities, in order to be able to find the influence of each factor in the process of college students ' Internet addiction.

- Internet addiction and the richness of college students ' network.

- Internet addiction of college students and learning.

- Network behavior and network addiction of college students. 


\section{Psychological intervention on Internet addiction of college students}

Psychological intervention refers to the application of psychological theories and techniques, help eliminate work, study and life caused by a variety of factors in cognitive confusion, emotional problems, depression and other psychological conditions, improve relationships, increase patient ability to adjust and adapt to the environment. Psychological counseling is designed to promote the patient's physical and mental health. Psychological intervention has been widely applied to health care, education and other fields, it is to help individuals to correct misconduct, ease bad feelings, maintain good mental health plays an important role. In view of the psychological intervention in practice and achieved good results, this method was gradually introduced to the intervention of Internet addiction in the past. In particular, the current, the external effectiveness of interventions to treat Internet addiction is not an obvious case, application of psychological intervention is particularly important.

Many scholars of psychological intervention techniques and methods used in the treatment of Internet addiction, summarized a lot of specific psychological interventions, according to its theory on the basis of different, there are several psychological interventions:

- Behavior therapy

- Psychoanalytic therapy

- Cognitive therapy

- Morita therapy

- Group counseling therapy

Psychological intervention on Internet addiction of college students.

(1) determine the intervention goals. Verify the effect of psychological intervention on Internet addiction of college students, revealed between college students ' mental health and relationships.

(2) the intervention of specific measures, including the option of intervening object, set the intervention period, determine the content of each intervention.

(3) the intervention process. In essence, Internet addiction is a psychological barrier, through the existing research findings and conclusions this investigation, Internet addiction of college students have a particular personality characteristics, such as low self-esteem, withdrawal, social disorder, and so on, the psychological intervention of Internet addiction, is to address the special adjustment and correction of the psychological characteristics, achieve the goal of successful treatment of Internet addiction.

(4) collect the intervention group and the control group before and after the intervention of the relevant data, on the basis of analysis of the data, determine the effect of the intervention.

\section{References}

[1]Davis RA,Flett GL.Validation of a New Seale of Measuring Problematic Internet Use: Implication for Pre-employment Screening. Cyber Psychology ehavior, 2007, 5(4).

[2]Chou.C, Hsiao M.Internet addiction usage, gratification and pleasure experience: the Taiwan college students'case. Computer\&Education. 2009,35(1):65-80.

[3]Young.K.S. Internet addiction: the emergence of an new clinical disorder. Cyber psychology and Behavior,2011,19(3):237-244.

[4]R.A.Davis. cognitive-behavior remodal of pathological internet use. Computers in Human Behavior ,2001,17(2):187-195.

[5]He Gongde. Japan take steps to keep teenagers away from network hazards. popular science and technology news, 2005.4.

[6]Wu Zengqiang, Zhang Jianguo. adolescent Internet addiction prevention and intervention [m]. Shanghai: Shanghai education press, 2007:19. 
[7]Yang Xiaofeng. Internet use and Internet addiction of college students and the relationship between mental health [d]. Inner Mongolia Normal University, 2012. 